Case Report

\title{
Melioidosis presenting as parotid abscess in children: two consecutive cases
}

\author{
KKMK Premaratne ${ }^{1}$, GKD Karunaratne ${ }^{1}$, R Dias $^{1}$, AK Lamahewage ${ }^{1}$, \\ M Samarasinghe ${ }^{1}$, E Corea $^{2}$, RMTM Gunawardena ${ }^{1}$ \\ Sri Lankan Journal of Infectious Diseases 2017 Vol.7 (2):112-118 \\ DOI: http://dx.doi.org/ 10.4038/sljid.v7i2.8149
}

\begin{abstract}
Melioidosis is an emerging infection in Sri Lanka with increasing case reports, mostly in adults but also in children. We report two children with parotid abscess, which is one of the common clinical manifestations in the paediatric population in Southeast Asia but rare in Northern Australia. Both were previously healthy. The children recovered completely after surgical drainage and antibiotic treatment according to standard guidelines. Because the spectrum of disease in childhood is different from that in adults and underlying predisposing factors are usually not seen in children, a high index of suspicion is required for the diagnosis of paediatric melioidosis. Raising awareness among paediatricians in Sri Lanka of the clinical presentations of melioidosis in childhood is a prerequisite for improved ascertainment. The key role of the microbiology laboratory in the diagnosis of melioidosis is highlighted.
\end{abstract}

Keywords: Melioidosis, Burkholderia pseudomallei, Parotid abscess, Children

\section{Introduction}

Melioidosis is an emerging tropical disease caused by the Gram negative bacillus, Burkholderia pseudomallei, which resides in soil and surface water. Inhalation of dust contaminated with the organism and percutaneous inoculation or ingestion of contaminated water are considered to be the main modes of transmission. ${ }^{1}$ The disease is most prevalent in Southeast Asia (SEA) and Northern Australia. It is probable that the endemic area extends beyond these two endemic regions as is evident from case reports from America and Africa. ${ }^{2}$ In Sri Lanka, an increasing number of cases are being reported, mostly in adults but also including a few paediatric cases. ${ }^{3}$ While clinical presentations are protean, ranging from acute sepsis to chronic infection, there is a distinct geographical variation with neurological melioidosis more common in Northern Australia and parotid abscess in children more common in Thailand. ${ }^{1}$ In this paper we report two patients admitted to the Lady Ridgeway Children's Hospital at the same time in 2016 with parotid abscesses.

\footnotetext{
${ }^{1}$ Lady Ridgeway Hospital for Children, Colombo 8, Sri Lanka

${ }^{2}$ Faculty of Medicine, University of Colombo, Sri Lanka

Address for correspondence: Dr KKMK Premaratne, Lady Ridgeway Hospital for Children, Colombo 8, Sri Lanka. Tel: +940714468198 Email: madarapremaratne@gmail.com (D)http://orcid.org/0000-0002-3666-9107
} 


\section{Case 1}

\section{Presenting Concerns}

A 9-year-old boy was transferred from the District General Hospital, Kilinochchi to Lady Ridgeway Children's Hospital, Colombo with a history of fever for 8 days and a painful parotid swelling on the right side of his face for 3 days. The swelling was gradually increasing in size. His past medical history was uneventful and his immunizations were up to date. Past surgical history was not significant except for surgery to evacuate a submental haematoma. Cultures were not done at that time and the patient was treated with oral antibiotics. He is the younger of two brothers and his father is a fisherman and mother, a housewife. He gave a history of frequent exposure to soil during playing. He usually bathes from a well and not from any natural water source. There is no history of travel outside Kilinochchi.

\section{Clinical Findings}

On examination he was febrile, with axillary temperatures of $101{ }^{\circ} \mathrm{F}-104{ }^{\circ} \mathrm{F}$, tachypnoea (respiratory rate of 48/min) and tachycardia (pulse rate of 102/min). A right sided tender parotid swelling of $4 \mathrm{~cm}$ was present. Other examination findings were normal. Blood investigation results on admission included a C-reactive protein (CRP) of $8.8 \mathrm{mg} / \mathrm{dl}$ and a white cell count of $7.09 \mathrm{x}$ $10^{9} / \mathrm{L}$ with $64.6 \%$ neutrophils. Ultrasound scan of the parotid swelling showed enlargement of the parotid gland on the right side with heterogenous echogenicity and multiple enlarged intraparotid lymph nodes, the largest being $3.2 \mathrm{~cm} \times 1.2 \mathrm{~cm}$. No abscess formation was noted. The clinical picture was compatible with acute on chronic parotitis with reactive lymphocytosis. The child was started on intravenous cefuroxime and metronidazole for two days but symptoms did not improve and co-amoxyclav was started after omitting the other antibiotics and cefotaxime added after two days.

\section{Diagnostic Focus and Assessment}

On day 3, incision and drainage of the parotid swelling was done and pus sent for culture. On direct Gram stain, no pus cells or organisms were seen. Pus was inoculated on blood, chocolate and MacConkey agar. On the following day, a Gram negative bacillus was isolated on all 3 plates. On blood agar, the colonies were $2-3 \mathrm{~mm}$ in size, beta-haemolytic and confluent areas had a metallic sheen. The isolate was oxidase positive. On MacConkey agar colonies were initially non lactose fermenting and 1-2 mm, round and convex but later became bright pink. The isolate was considered to be a Pseudomonas species and sensitivity testing was done accordingly. A tube of Kligler iron agar (KIA) was inoculated. 


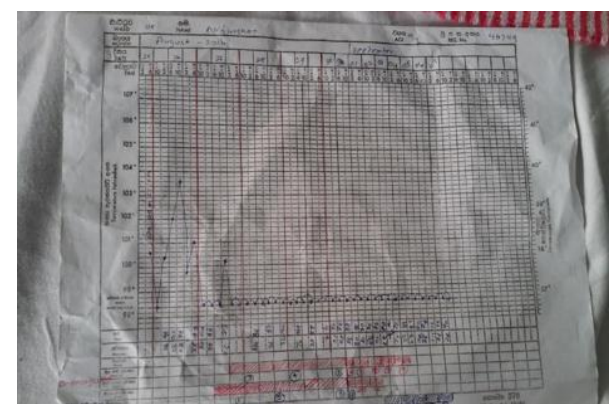

Figure 1. Temperature chart of Case 1

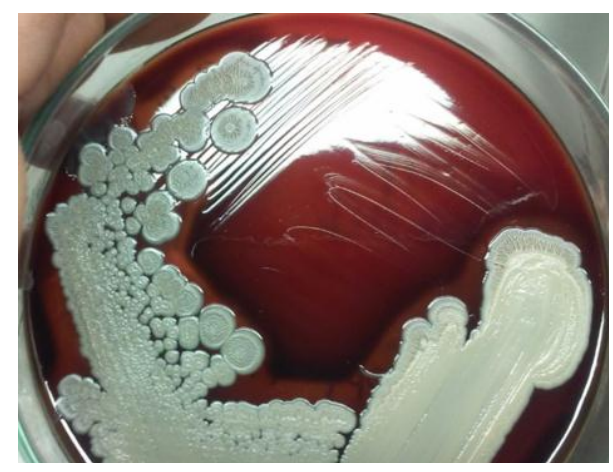

Figure 2. B. pseudomallei on blood agar after 5 days of incubation at $37{ }^{\circ} \mathrm{C}$

\section{Therapeutic Focus and Assessment}

The clinicians were informed and advised to change the antibiotic to ceftazidime. As the child had improved after abscess drainage and fever had reduced (Figure 1) the clinical staff were reluctant to change antibiotics despite continued parotid swelling. On reading the antibiotic sensitivity test it was noted that the isolate was sensitive to ceftazidime, meropenem and imipenem but resistant to all the aminoglycosides (gentamicin, amikacin and netilmicin). The KIA tube was non-reactive. Due to these readings and the suspicious colony morphology on blood agar (Figure 2) and MacConkey agar, it was suspected that the isolate was B. pseudomallei and Gram stain was performed. The 'safety pin'-like appearance of the isolate further strengthened this possibility. On further sensitivity testing the isolate was found to be sensitive to co-amoxyclav and co-trimoxazole and resistant to colistin, typical of B. pseudomallei.

The clinicians were informed of the possibility of melioidosis and again advised to change the antibiotics to ceftazidime. The isolate was sent to the Department of Microbiology, Faculty of Medicine, University of Colombo for confirmation of identity by latex agglutination and PCR testing. Blood from the patient was sent to detect antibodies to B. pseudomallei. The isolate was confirmed as B. pseudomallei and the child's melioidosis antibody titer by the indirect haemagglutination assay was 160 .

The boy was treated with ceftazidime for two weeks in the intensive phase, and was discharged on oral cotrimoxazole and folic acid for 3 months as eradication therapy. His mother was educated on the infection including the importance of continuing oral antibiotics and follow up to prevent relapse.

\section{Case 2}

\section{Presenting Concerns}

A 9-year-old girl was transferred from District General Hospital, Chilaw to the Lady Ridgeway Children's Hospital, Colombo, with a history of fever for 10 days, pain in the left ear and left-sided facial swelling of nearly two weeks duration. Her past medical and surgical history was uneventful except that she had under gone a nasal polypectomy when she was 5 years old. Immunization was up to date. She was the second child in a family of three children. Her father was a driver of heavy vehicles and her mother, a housewife. Her mother engages in home gardening of vegetables and chillies and she often helps her mother in gardening. Water from a tube well is used for drinking 
and bathing. There was no history of travel outside Chilaw. She participates in sports events, especially athletics.

\section{Clinical Findings}

On examination she was febrile (axillary temperature of $104^{\circ} \mathrm{F}$ ) and tachypnoea (respiratory rate of $34 / \mathrm{min}$ ) and tachycardia (pulse rate of $100 / \mathrm{min}$ ) were noted. There was a tender swelling of the parotid on the left side with a discharge from a previous needle aspiration site. There was mild left-sided facial palsy with the mouth slightly deviated to the right side. Her CRP was elevated ranging from $59.7-238 \mathrm{mg} / \mathrm{dl}$ and her white cell count was $26.6 \times 10^{9} / \mathrm{L}$ with $80 \%$ neutrophils. Ultrasound scan of the parotid swelling showed enlargement of the left parotid gland compatible with acute parotitis but no abscess formation was noted.

\section{Diagnostic Focus and Assessment}

Needle aspiration of the parotid lump was done twice and she was started on intravenous

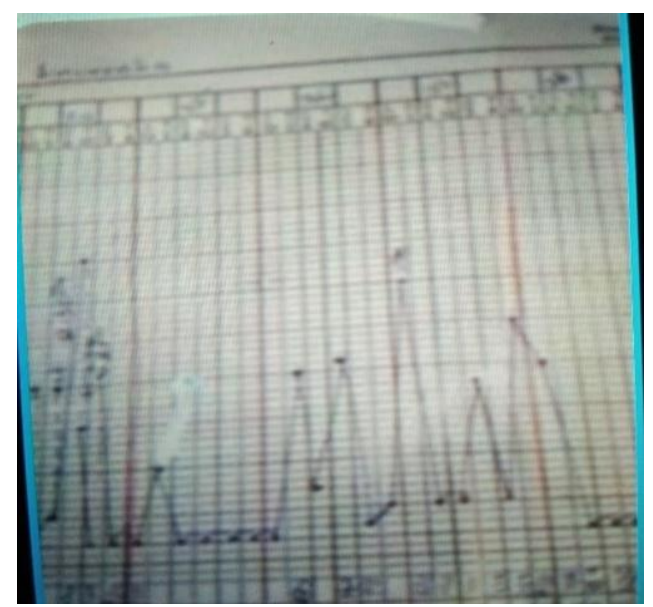

Figure 3. Temperature chart of Case 2 cefuroxime and metronidazole. Cefuroxime was omitted after 4 days and co-amoxyclav was substituted. Incision and drainage of the parotid swelling was done and pus was sent to the microbiology laboratory. At that time, with Case 1 in mind, the possibility of melioidosis was considered and the clinicians were contacted and advised to change the antibiotic to ceftazidime as the child continued to have fever spikes despite surgery (Figure 3). Blood was also taken for melioidosis antibodies.

Results of pus examination in the microbiology laboratory was similar to Case 1. Melioidosis antibody titer was 320.

\section{Therapeutic Focus and Assessment}

The patient was treated with the same regimen as Case 1.

\section{Discussion}

We report two paediatric cases of melioidosis presenting with parotid abscess. Both were 9 years old, one male and one female. Neither of the children had any predisposing factors but both had frequent contact with soil. One case was from Kilinochchi in the Northern Province of Sri Lanka while the second was from Chilaw in the North Western Province. Both presented with high fever spikes but only one had an increased leucocyte count with neutrophil predominance and increased CRP levels.

Melioidosis is more common in adults than in children and infections in children represent only 5$15 \%$ of all cases. ${ }^{1,4}$ Clinical manifestations also differ between the two groups. Pneumonia is the most frequent presentation in adults and bacteraemia is also common. Predisposing risk factors like diabetes mellitus, chronic renal failure, chronic lung and liver disease are commonly (60\%- 
$90 \%$ ) associated with adult patients. In contrast, bacteraemia is less common in children, who often present with skin and soft tissue abscesses, with most having no predisposing factors. ${ }^{5}$

There is a distinct geographical variation in the clinical presentation in children, with suppurative parotitis more common in SEA and skin and soft tissue abscesses (other than parotid abscess) reported from Northern Australia. ${ }^{1}$ The reason for the greater occurrence of parotid involvement in SEA is explained as probably due to the consumption of unchlorinated water from natural sources $^{6}$ or to genetic variations in the strains of B. pseudomallei. $^{7}$

Abscess formation, spontaneous rupture into the auditory canal, facial nerve palsy, septicaemia, osteomyelitis and necrotizing fasciitis are reported as complications of melioidosis parotitis. ${ }^{8,9}$ One of our patients developed a palsy of the marginal mandibular branch of the left facial nerve which improved spontaneously.

The causative organism of melioidosis, B. pseudomallei, is a soil saprophyte with the ability to become a pathogen of both human and animals. It is an aerobic, non-spore forming, motile, Gram negative bacillus with varying colony morphology. Initially smooth colonies may become wrinkled with further incubation at $37{ }^{\circ} \mathrm{C}$ or room temperature, as shown in one of our isolates (Figure 1).

With regard to laboratory diagnosis, the gold standard is isolation of the organism from blood or other body fluids of the patient. Although $B$. pseudomallei is not a fastidious organism and grows readily in routine laboratory culture media such as blood agar and MacConkey agar, even pure growths are often missed as it is often misidentified. As it is oxidase positive, it is mostly misreported as Pseudomonas species. However, it may sometimes be reported as a coliform due to the bright pink colonies seen on MacConkey agar after 48 hours incubation. A high degree of awareness and familiarity among laboratory staff regarding the colony morphology and the culture characteristics of the organism is therefore needed. Preliminary identification is often made on the grounds of the Gram stain showing the typical 'safety pin' appearance and by demonstrating résistance to gentamicin and other aminoglycosides and colistin or polymyxin B and sensitivity to amoxicillin-clavulanic acid and trimethoprim-sulfamethoxazole. In our first case we considered the isolate as a Pseudomonas species until we noticed the aminoglycoside resistance of the organism.

Confirmation of the identity of the isolate can be difficult. Commercial identification kits such as API $20 \mathrm{NE}$ and Rap Id NF often give incorrect results, identifying the isolate as Burkholderia cepacia. ${ }^{10}$ The latex agglutination test has been shown to demonstrate high accuracy to confirm the identity of the culture isolate. ${ }^{11}$ Confirmation can also be done by PCR. ${ }^{12}$ The latter two tests are available in Sri Lanka. Rapid diagnosis on patients' specimens using PCR and direct immunofluorescence have been found to be less sensitive than the culture. ${ }^{13,14}$ Antigen detection using a lateral flow immunoassay is a recent development and is expected to give reliable results in pus but not in blood. ${ }^{15}$ Unfortunately, these tests are only performed in reference laboratories and are not available in clinical laboratories.

Presumptive diagnosis using serological tests can be made in both the systemic and localized form of melioidosis as $B$. pseudomallei is capable of producing a humoral immune response in the host. False positives can be seen in the presence of subclinical and asymptomatic infections and also 
due to cross-reacting antibodies from other organisms. ${ }^{16}$ Melioidosis antibody tests were done in both our cases using the indirect haemagglutination assay and was positive at slightly elevated titres in both.

According to the Darwin Melioidosis Guidelines ${ }^{17}$, treatment is divided into two phases, the intensive phase and the eradication phase. The drug of choice for the intensive phase is intravenous ceftazidime but for patients in the intensive care unit or with neurological disease, meropenem can be considered. Oral trimethoprim-sulfamethoxazole for 3-5 months is recommended for the eradication phase. Follow up is important as relapse rates are high if the patient is less compliant during the eradication phase. Some recent case reports of localized melioidosis in children demonstrated successful outcomes without relapses after treating the intensive phase with oral antibiotics. ${ }^{5}$ Both patients in our study were transferred back to the referring hospitals and were lost to follow up.

\section{Conclusion}

Clinical diagnosis of melioidosis in children is often more difficult than in adults because of lack of awareness among paediatricians and because children do not have the common predisposing factors. Therefore, it is important to have a high index of suspicion of melioidosis in any fever of unknown origin or skin and soft tissue infection, especially parotid abscess, as it is the commonest presentation in the Southeast Asian region. The microbiology laboratory will continue to play a key role in diagnosing and managing melioidosis.

\section{References}

1. Currie BJ, Ward L, Cheng AC. The epidemiology and clinical spectrum of melioidosis: 540 cases from the 20 year Darwin prospective study. PLoS Negl Trop Dis. 2010; 4:e900 doi : http://dx.doi.org/10.1371/journal.pntd.0000900

2. Currie BJ, Dance DA, Cheng AC. The global distribution of Burkholderia pseudomallei and melioidosis: an update. Trans R Soc Trop Med Hyg 2008; 102 (suppl 1):S1-4 doi : http://dx.doi.org/10.1016/S0035-9203(08)70002-6

3. Corea EM, Merritt AJ, Ler Y-H, et al. Sri Lankan National Melioidosis Surveillance Program uncovers a nationwide distribution of invasive melioidosis. The American Journal of Tropical Medicine and Hygiene. 2016; 94(2):292-298. doi : http://dx.doi.org/10.4269/ajtmh.15-0567

4. Dance DAB, Davis TME, Wattanagoon Y, et al. Acute suppurative parotitis caused by Pseudomonas pseudomallei in children. J infect Dis. 1989; 159:654-660. doi : http://dx.doi.org/10.1093/infdis/159.4.654

5. McLeod C, Morris PS, Bauert PA, et al. Clinical presentation and medical management of melioidosis in children: A 24- Year prospective study in the Northern Territory of Australia and review of the literature Clinical Infectious Diseases 2015; 60(1):21-6. doi : http://dx.doi.org/10.1093/cid/ciu733

6. Limmathurotsakul D, Kanoksil M, Wuthiekanun V, et al. Activities of daily living associated with acquisition of melioidosis in northeast Thailand: a matched case control study. PLoS Negl Trop Dis. 2013; 7:e2072

doi : http://dx.doi.org/10.1371/journal.pntd.0002072 
7. Pearson T, Giffard P, Beckstrom-Sternberg S, et al. Phylogeographic reconstruction of a bacterial species with high levels of lateral gene transfer. BMC Biol. 2009; 7:78.

doi : http://dx.doi.org/10.1186/1741-7007-7-78

8. Lim WK, Gurdeep GS, Norain K. Melioidosis of the head and neck. Med J Malaysia. 2001; 56(4):471-7. No doi

9. Shivbalan S, Reddy N, Tiru V, Thomas K. Systemic melioidosis presenting as suppurative parotitis. Indian Pediatr. 2010; 47(9):799-801.

doi : http://dx.doi.org/10.1007/s13312-010-0106-2

10. Glass MB, Popovic T. Preliminary evaluation of the API 20NE and RapID NF Plus systems for rapid identification of Burkholderia pseudomallei and B. mallei. Journal of Clinical Microbiology. 2005; 43(1):479-483.

doi : http://dx.doi.org/10.1128/JCM.43.1.479-483.2005

11. Duval BD, Elrod MG, Gee JE, et al. Evaluation of a Latex Agglutination Assay for the identification of Burkholderia pseudomallei and Burkholderia mallei. The American Journal of Tropical Medicine and Hygiene. 2014; 90(6):1043-1046. doi : http://dx.doi.org/10.4269/ajtmh.14-0025

12. Merritt A, Inglis TJJ, Chidlow G, Harnett G. PCR-based identification of Burkholderia pseudomallei. Rev. Inst. Med. Trop. São Paulo. 2006; 48(5):239-244. doi : http://dx.doi.org/10.1590/S0036-46652006000500001

13. Wiersinga WJ, Currie BJ, Peacock SJ. Melioidosis. N Engl J Med. 2012; 367:1035-44. doi : http://dx.doi.org/10.1056/NEJMra1204699

14. Wuthiekanun V, Desakorn V, Wongsuvan g et al, Rapid immunofluorescence microscopy for diagnosis of melioidosis. Clin Diagn Lab Immunol. 2005; 12:555-6 doi : http://dx.doi.org/10.1128/CDLI.12.4.555-556.2005

15 Houghton RL, Reed DE, Hubbard MA, et al. Development of a Prototype Lateral Flow Immunoassay (LFI) for the rapid diagnosis of melioidosis . PLOS Neglected Tropical Diseases. 2014;8(3): e2727 doi : http://dx.doi.org/10.1371/journal.pntd.0002727

16. Vadivelu J, Puthucheary SD, Gendeh GS, Parasakthi N. Serodiagnosis of melioidosis in Malaysia. Singapore Med J. 1995; 36:299-302. No doi

17 Pitman MC, Luck T, Marshall CS, et al. Intravenous therapy duration and outcomes in melioidosis: A new treatment paradigm. PLOS Neglected Tropical Diseases. 2015; 9(3): e0003586. doi : http://dx.doi.org/10.1371/journal.pntd.0003586 